\title{
Complicación orbitaria recurrente por rinosinusitis. Reporte de un caso
} Recurrent orbital complication by rhinosinusitis. A case report

\author{
Dra. Roxana Spini ${ }^{a}$ Dr. Daniel Cruz ${ }^{a}$, Dra. Lucía Fernández $z^{a}$ y Dr. Federico Urquiola ${ }^{b}$
}

\section{RESUMEN}

El compromiso orbitario es la complicación más frecuente de la rinosinusitis aguda en pediatría, y el etmoides es el seno más afectado. La recurrencia es infrecuente. Existen solo tres casos publicados en la literatura. Una anomalía anatómica del proceso unciforme o un mucocele podrían ser factores predisponentes, que pueden obstruir el drenaje normal de los senos junto con la presencia de etmoiditis.

La tomografía computada y la resonancia magnética orientan el diagnóstico. El tratamiento quirúrgico está indicado ante la falta de respuesta al tratamiento instaurado luego de 48 horas o disminución de la agudeza visual o recurrencias sin patología tumoral de base.

Se presenta a un paciente de 4 años de edad que consultó por un cuadro compatible con etmoiditis complicada recurrente. Presentó celulitis preseptal en seis oportunidades y un episodio de absceso subperióstico, desde los 3 meses de edad. Evolucionó favorablemente luego de la operación quirúrgica.

Palabras clave: celulitis orbitaria, celulitis preseptal recurrente, niños, sinusitis, sinusitis del etmoides.

\begin{abstract}
Orbital infection is the most frequent complication of ethmoiditis. Recurrent periorbital cellulitis is a very rare complication of rhinosinusitis with only three reports in the literature describing this pathological process.

This complication can be favored by an anatomical abnormality of the uncinate process or mucocele obstructing the normal drainage pathway, in addition to ethmoidal sinusitis.

Computed tomography and magnetic resonance guide the diagnosis. The treatment is based on antibiotics, corticosteroids and local decongestants. Surgical treatment is indicated in the absence of response to treatment established after 48 hours or decrease in visual acuity or recurrent orbital complications without underlying tumor pathology.
\end{abstract}

a. División de Otorrinolaringología del Hospital General de Niños Pedro de Elizalde, Ciudad Autónoma de Buenos Aires.

b. Servicio de Otorrinolaringología del Hospital Italiano de Buenos Aires, Ciudad Autónoma de Buenos Aires.

Correspondencia:

Dra. Roxana Spini: roxanaspini@intramed.net

Financiamiento: Ninguno.

Conflicto de intereses: Ninguno que declarar.

Recibido: 7-1-2019

Aceptado: 2-7-2019
In this report, we present a case of orbital complication of rhinosinusitis in a 4 year-old-child with six episodes of unilateral periorbital cellulitis and one episode of subperiosteal orbital abscess since the age of 3 months. There was a complete resolution with no recurrence after the surgical intervention. Key words: orbital cellulitis, recurrent periorbital cellulitis, ethmoid sinusitis, children, sinusitis.

http: / / dx.doi.org/10.5546/aap.2019.e670

Cómo citar: Spini R, Cruz D, Fernández L, Urquiola F. Complicación orbitaria recurrente por rinosinusitis. Reporte de un caso. Arch Argent Pediatr 2019;117(6):e670-e675.

\section{INTRODUCCIÓN}

La complicación más frecuente de rinosinusitis en pediatría es la orbitaria. La celulitis preseptal recurrente o, en su defecto, la complicación orbitaria recurrente (COR) es muy poco frecuente. Durante los procesos inflamatorios agudos, como la rinitis, está descrito que hay factores predisponentes que ocasionan un compromiso en el mecanismo de drenaje mucociliar, una anomalía anatómica del proceso unciforme o un mucocele, que favorece que se perpetúen las COR. ${ }^{1}$

El tratamiento quirúrgico está indicado si falla el tratamiento médico luego de 48 horas, si hay disminución de la agudeza visual o recurrencias sin patología tumoral de base.

El objetivo es presentar un caso clínico de celulitis preseptal recurrente en un paciente pediátrico que requirió una operación quirúrgica para su resolución.

\section{CASO CLÍNICO}

Se presenta a un paciente de 4 años que tuvo seis episodios de celulitis preseptal y un episodio de absceso subperióstico en toda su evolución (cinco fueron derechas y dos, izquierdas). El primer episodio fue a los tres meses de vida.

Los primeros episodios fueron tratados en otro hospital y, a partir del cuarto, se inició el seguimiento en nuestro Servicio por celulitis preseptal derecha. Al momento del examen físico (Figura 1), presentaba fiebre, edema, eritema y flogosis en la región periorbitaria, exoftalmos, 
con dolor ante la movilización ocular, por lo que se solicitó una tomografía computada (TC) del macizo facial, que informó (Figura 2) órbita derecha: masa en contacto con lámina papirácea que desplazaba lateralmente el recto interno compatible con absceso retroorbitrario (subperióstico); engrosamiento de la mucosa de los senos maxilares; ocupación parcial del etmoides izquierdo y total del etmoides derecho y del esfenoides con cavum libre. Permaneció internado y cumplió 18 días de tratamiento endovenoso con cefriaxona (50 $\mathrm{mg} / \mathrm{kg} /$ día),

FIGURA 1. Examen físico: edema y eritema de la región periorbitaria derecha. Leve exoftalmos
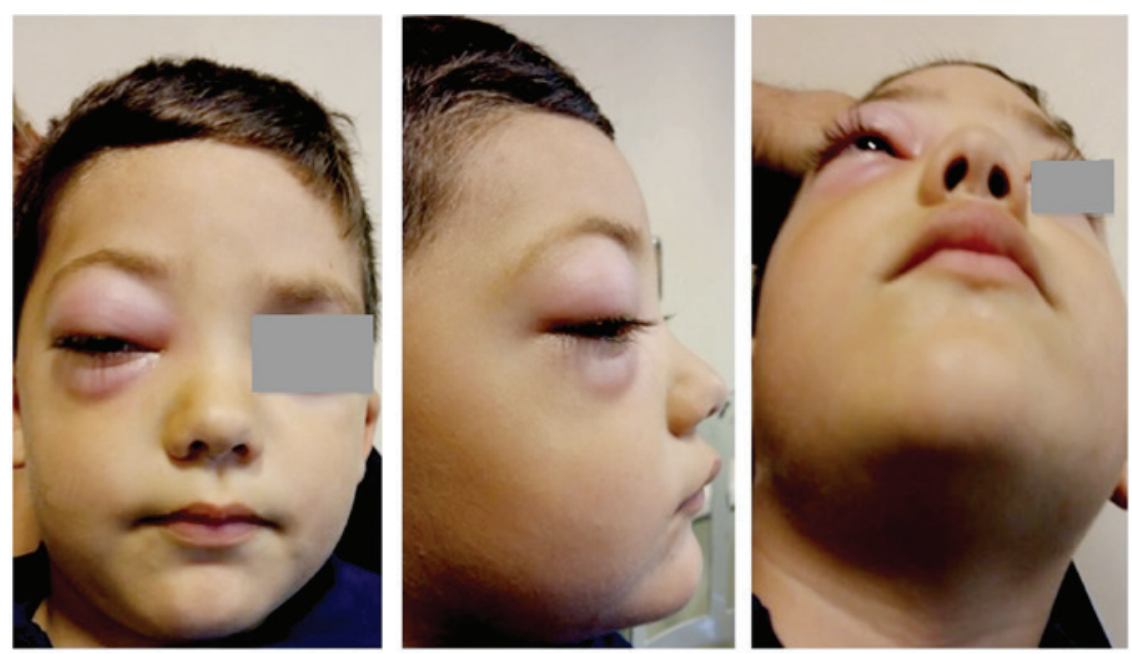

FiguRA 2. A. Corte axial: presencia de masa en contacto con la lámina papirácea que desplaza lateralmente el recto interno compatible con absceso retroorbitrario (subperióstico). Ocupación total del etmoides derecho. B. Corte axial: ocupación parcial del etmoides izquierdo. C. Corte axial: engrosamiento mucoso de ambos senos maxilares. D. Corte coronal: se visualiza la ocupación completa del etmoides derecho
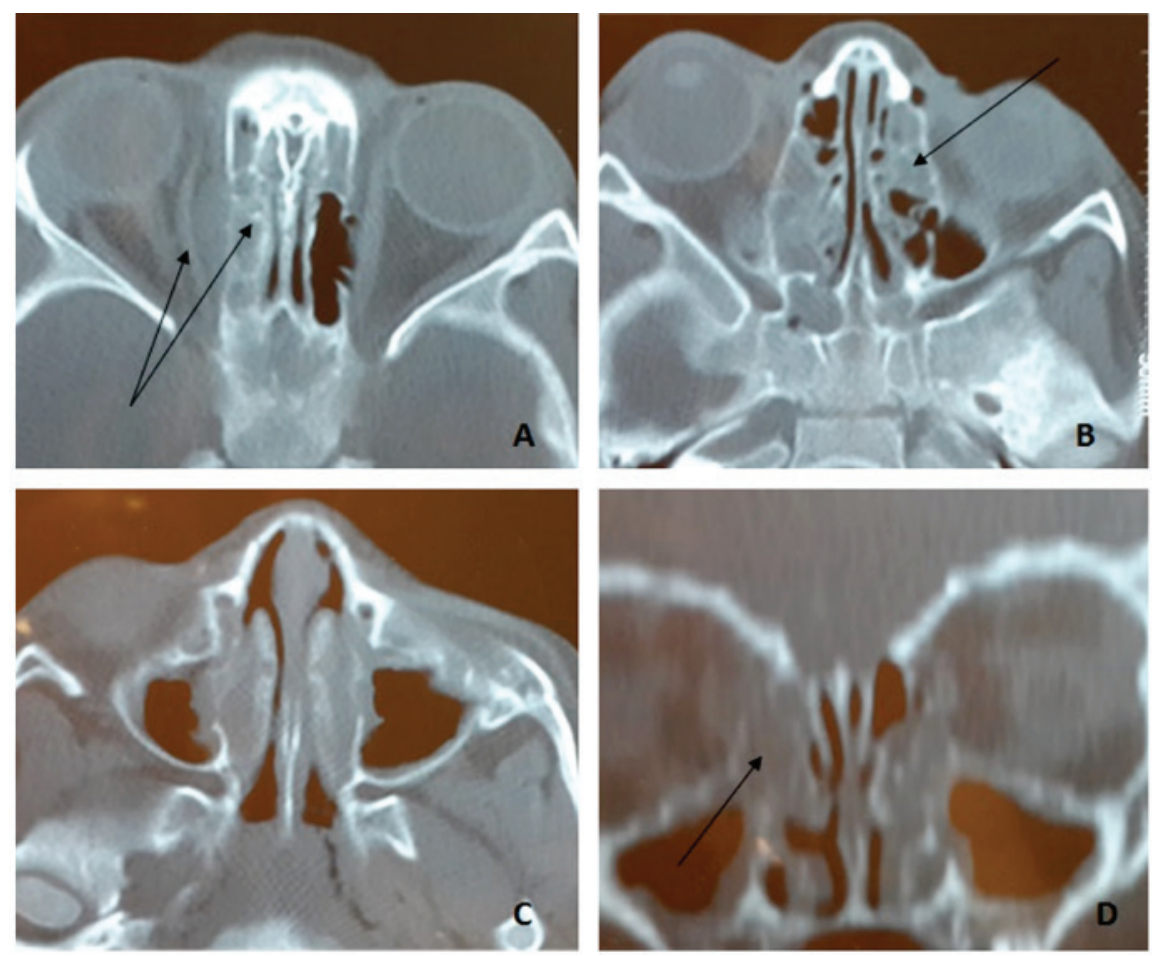
clindamicina (30 mg/ kg/ día) y dexametasona $(0,6 \mathrm{mg} / \mathrm{kg} /$ día $)$, con buena respuesta. Se solicitó una resonancia magnética nuclear (RMN) del macizo facial con contraste para realizar en forma ambulatoria.

Se inició un manejo multidisciplinario con los Servicios de Alergia, Inmunología, Oftalmología e Infectología. Se realizaron serologías para hepatitis $B, A$, rubéola y virus de la inmunodeficiencia humana $(\mathrm{VIH})$, con resultado negativo. Se solicitaron inmunoglobulinas A (IgA), M (IgM), G (IgG), CD3, CD4 y subtipos de IgG normal. Perfil reumatológico normal. Oftalmología descartó compromiso ocular y defecto anatómico que pudiera predisponer a dicha patología. El Servicio de Alergia descartó algún factor predisponente.

La RMN demoró 4 meses debido a la demanda y la poca accesibilidad del municipio. Durante ese lapso, el paciente repitió 3 episodios más de celulitis preseptal, dos derechas y una izquierda, y una de ellas (6. ${ }^{\text {to }}$ episodio) requirió una nueva internación (Figura 3). El tratamiento en todos los episodios consistió en 21 días de antibiótico, con buena respuesta. En la internación, se indicó la combinación de ceftriaxona (50 mg/ kg/día), clindamicina (30 mg/ kg/día) y dexametasona (0,6 mg $/ \mathrm{kg} /$ día $)$ y, en forma ambulatoria, la combinación de trimetoprima-sulfametoxazol (10 mg/kg/día), amoxicilina-ácido clavulánico
(50 mg/ kg/ día) y betametasona (1 mg/ $\mathrm{kg} /$ dosis). El informe de la RMN en secuencia T1 y T2 (Figura 4) mostró la ocupación del etmoides posterior derecho por material hipointenso, heterogéneo, sin compromiso de las estructuras vecinas. Por lo que, fuera de una intercurrencia aguda, se decidió la operación quirúrgica para la exploración y el tratamiento. Se observó un engrosamiento cartilaginoso del septum en forma bilateral, que dificultó el abordaje al meato medio. Se realizó una etmoidectomía anterior y posterior por vía endoscópica derecha, en la que se observó un edema generalizado de celdas etmoidales con presencia de masa homogénea de color parduzco de aproximadamente $0,5 \mathrm{~cm} \times 1,5 \mathrm{~cm}$ en el etmoides posterior y que se envió a Anatomía Patológica (Figura 4 D). Uncinectomía en forma bilateral; se extrajo del complejo osteomeatal (COM) izquierdo una masa homogénea blanco grisáceo de consistencia blanda compatible con un pólipo. Resultado de Anatomía Patológica: inflamación crónica, congestión y edema. Procedimiento sin complicaciones con favorable evolución posoperatoria. No presentó recurrencia luego de 2 años del procedimiento quirúrgico.

\section{DISCUSIÓN}

El compromiso orbitario es la complicación más frecuente de la rinosinusitis aguda en pediatría (el 74-85\%), y el etmoides es el seno con
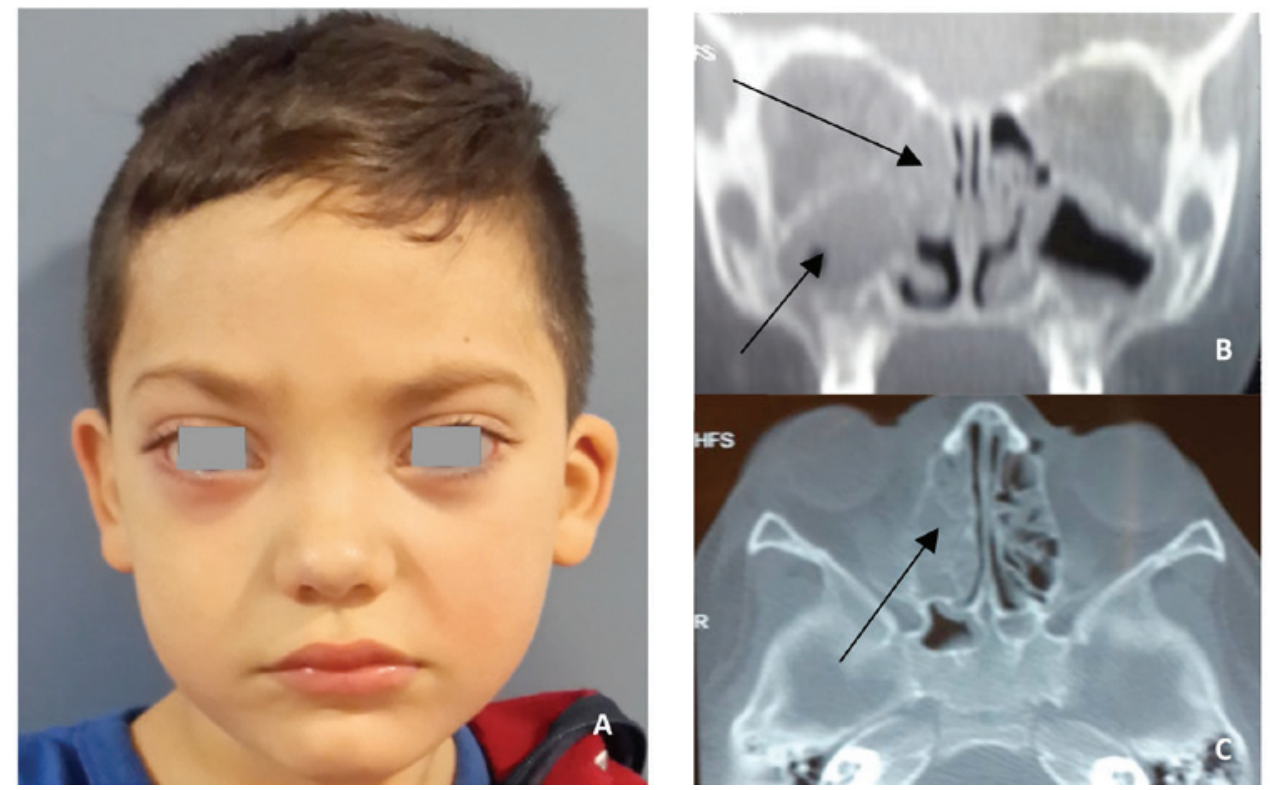
mayor compromiso. ${ }^{2}$ La diseminación se produce por vía ósea, venosa o linfática, favorecida por la existencia de comunicaciones entre las celdillas etmoidales y la órbita. La osteítis de la lámina papirácea se encuentra asociada con complicaciones orbitarias por rinosinusitis, debido al hueso inmaduro y a la mayor vascularización en la población pediátrica..$^{2-6}$

La edad media de presentación de la etmoiditis es entre los 3 y los 5 años. ${ }^{7}$ En una serie de 42 niños, publicada por Brown et al., ${ }^{7}$ la media de edad era de 5 años para los niños que presentaban celulitis preseptal y de 11 años para los que el compromiso era retroseptal. Existen casos publicados de complicaciones orbitarias en neonatos. 8,9

Los microorganismos causantes, en orden de frecuencia, pueden ser aerobios (Streptococcus pneumoniae, Staphylococcus aureus) y anaerobios. Sin embargo, algunos autores ${ }^{5,10}$ encontraron un rescate mayor de Staphylococcus aureus. No es recomendable la toma de muestra de secreciones endonasales por carecer de esterilidad. ${ }^{5} \mathrm{El}$ rescate microbiológico es bajo, dada la instauración de antibióticos antes de la toma de estos. ${ }^{6,7}$ Botting y col., ${ }^{4}$ observaron que el $31 \%$ de los casos con compromiso retroseptal obtuvieron rescate positivo y solo el $23 \%$ de los pacientes con compromiso preseptal.

El diagnóstico es clínico. La TC del macizo facial y órbita con contraste confirma la sinusitis etmoidal, el compromiso de otros senos y descarta una complicación intraorbitaria o intracraneal. Ante la sospecha de trombosis del seno cavernoso, se debe solicitar una RMN, ya que la TC no la descarta.,11

El tratamiento consiste en antibióticos, corticoides y descongestivos locales. Se indica el manejo ambulatorio con amoxicilina-clavulánico (50 mg/ kg/día) y seguimiento estricto ante buen estado general y medio confiable. De lo contrario, se interna al paciente para el tratamiento antibiótico endovenoso con ceftriaxona $(50 \mathrm{mg} /$ $\mathrm{kg} /$ día) asociada a metronidazol (15 mg/ $\mathrm{kg} /$ día) o clindamicina (30 mg/ $\mathrm{kg} /$ día). Se sugiere el tratamiento endovenoso como mínimo 5 días y completar las 3 semanas por vía oral. ${ }^{5,7}$

Figura 4. Resonancia magnética nuclear. A, B, C. Secuencia T1, ocupación del etmoides posterior derecho por material hipointenso, heterogéneo sin compromiso de las estructuras vecinas (véase la flecha). D. Masa homogénea de color parduzco en el etmoides posterior. Resultado de Anatomía Patológica: inflamación crónica, congestión y edema
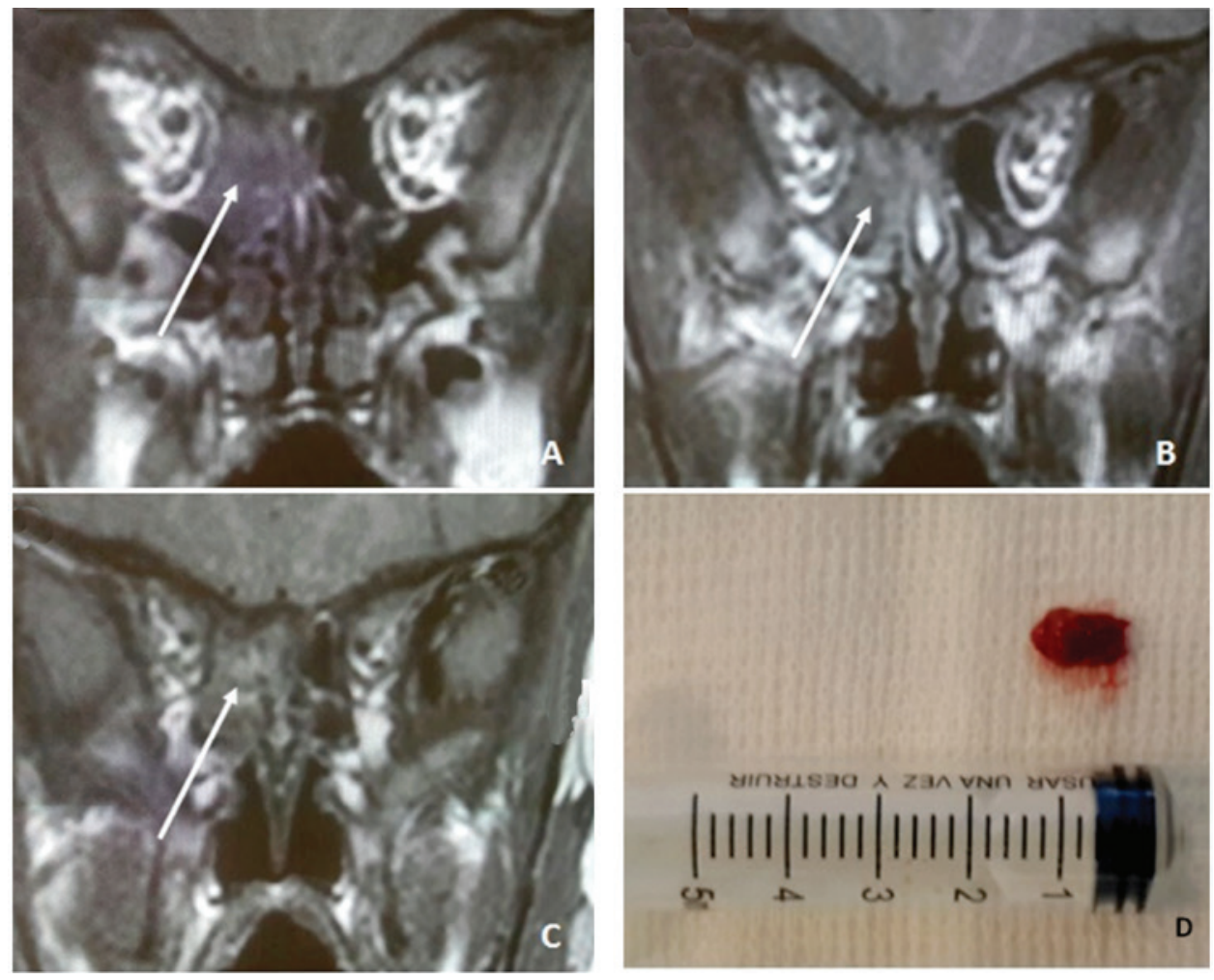
Ante la falla del tratamiento luego de 48 horas o la disminución de la agudeza visual, se debe realizar una exploración quirúrgica que permita el drenaje de abscesos y la descompresión orbitaria. Consiste, según el caso, en etmoidectomía, uncinectomía, antrostomía y / o drenaje del absceso por vía endoscópica y / o externa. ${ }^{4,5,7,11}$

La COR debe advertir una causa predisponente: variante anatómica o de un mucocele. Los mucoceles etmoidales se manifiestan en la TC como un adelgazamiento de las paredes de una celdilla etmoidal, con erosión ósea sin lisis, con un contenido homogéneo y sin realce tras la inyección de contraste. Las variantes anatómicas se pueden visualizar en la TC, luego del episodio infeccioso, o bien por visualización directa en la cirugía. ${ }^{12}$ Se deben tener en cuenta infecciones, como cisticercosis orbitaria, infección por VIH; trastornos hereditarios, como el síndrome de Shwachman-Diamond, y tumores orbitarios (sarcoma, linfangioma quístico o la localización orbitaria de una leucosis), que pueden revelarse por un exoftalmos rápido con signos inflamatorios o sobreinfección. Estos últimos presentan una mejora parcial con el tratamiento antibiótico con la presencia de exoftalmos persistente. ${ }^{7,11-13}$

Hay solo tres casos publicados en la literatura de COR. ${ }^{1,13,14}$ Karkos describió a un niño de 5 años que presentó, desde los dos años, 6 episodios de celulitis preseptal y un absceso subperióstico. Se realizó una cirugía endoscópica y se encontró el proceso unciforme largo y curvo que causaba la obstrucción del COM.

Jatana et al., presentaron a un niño de 28 meses de edad con celulitis preseptal unilateral recurrente desde los 11 meses de edad. Inicialmente, fue sometido solo a adenoidectomía, pero, con la séptima recurrencia, se realizó uncinectomía, antrostomía y etmoidectomía anterior. En la cirugía, se halló un proceso unciforme anormal en continuidad con la bulla etmoidal.

Caulley et al. presentaron a un niño de 4 meses con COR con afectación alternante de cada ojo. En el cuarto episodio, fue tratado como una osteomielitis, con buen resultado y sin recidivas.

Nuestro paciente se asemeja al paciente publicado por Karkos en la edad, en la cronología de los procesos infecciosos y en la resolución quirúrgica. Pero, en nuestro caso, los procesos infecciosos fueron derechos e izquierdos en forma alternante.
Es interesante que, en dos casos publicados de COR y en nuestro paciente, se observó una anomalía anatómica del proceso unciforme o una masa inflamatoria (en nuestro caso) que obstruía la vía de drenaje normal, además de la etmoiditis. La COR debe alertar sobre la posibilidad de variantes anatómicas o de tumoraciones inflamatorias o neoplásicas como causa predisponente.

En el paciente descrito, por los hallazgos observados en el momento de la cirugía y la conducta quirúrgica empleada, se considera que, al aumentar la permeabilidad del COM, mejoró la ventilación maxilar y etmoidal, y se evitó, así, la recurrencia. Dada la corta edad del paciente, se decidió la posible septumplastia para un segundo tiempo en caso de ser necesario.

\section{REFERENCIAS}

1. Karkos PD, Karagama Y, Karkanevatos A, Srinivasan V. Recurrent periorbital cellulitis in a child. A random event or an underlying anatomical abnormality? Int J Pediatr Otorhinolaryngol. 2004; 68(12):1529-32.

2. Nocon C, Baroody F. Acute Rhinosinusitis in Children. Curr Allergy Asthma Rep. 2014; 14(6):443.

3. Mañás Pujol M, Cisa Lluís E, Cruellos Taissik F. Complicaciones de las sinusitis. En Suarez Nieto C, Gil-Carcedo García L, Marco Algara J, et al. Tratado de otorrinolaringología y cirugía de cabeza y cuello. 2. ${ }^{\text {a }}$ ed. Tomo I. Buenos Aires: Panamericana; 2007.Págs.765-79.

4. Suhaili DN, Goh BS, Gendeh BS. A ten year retrospective review of orbital complications secondary to acute sinusitis in children. Med J Malaysia. 2010; 65(1):49-52.

5. Botting A, McIntosh D, Mahadevan M. Paediatric preand post-septal peri-orbital infections are different diseases. A retrospective review of 262 cases. Int J Pediatr Otorhinolaryngol. 2008; 72(3):377-83.

6. Brown CL, Graham SM, Griffin MC, Smith RJ, et al. Pediatric medial subperiosteal orbital abscess: medical management where possible. Am J Rhinol. 2004; 18(5):312-7.

7. Eviatar E, Sandbank J, Kleid S, Gavriel H. The role of osteitis of the lamina papyracea in the formation of subperiosteal orbital abscess in young children. Int J Pediatr Otorhinolaryngol. 2014; 78(12):2267-70.

8. Murray A, Albanasawy L, Morrissey MS. Periorbital cellulitis secondary to ethmoiditis in a 5-week-old child. Int J Pediatr Otorhinolaryngol. 2000; 52(1):101-3.

9. Rogers GA, Naseri I, Sobol SE. Methicillin-resistant Staphylococcus aureus orbital abscess in a neonate. Int J Pediatr Otorhinolaryngol. 2007; 71(6):991.

10. Coudert A, Ayari-KhalfallahS, Suy P, Truy E. Microbiology and antibiotic therapy of subperiosteal orbital abscess in children with acuteethmoiditis. Int J Pediatr Otorhinolaryngol. 2018; 106:91-5.

11. Sorin A, April M, Ward R. Recurrent periorbital cellulitis: an unusual clinical entity. Otolaryngol Head Neck Surg. 2006; 134(1):153-6.

12. Galindo-Ferreiro A, Alkatan H, ElKhamary S, AlDosairi S, Cruz AAV. Recurrent orbital inflammation mimicking orbital cellulitis associated with orbitopalpebral venous lymphatic malformation. Ophthalmic Plast Reconstr Surg. 2017; 33(5):e112-4. 
13. Jatana KR, Grischkan JM, Skomoroski MJ, Elmaraghy CA. Recurrent unilateral periorbital cellulitis in a pediatric patient-an anatomic abnormality. Int J Pediatr Otorhinolaryngol. 2008; 72(10):1577-80.
14. Caulley L, Corsten G,Hong P. Recurrent periorbital cellulitis in a very young child: a case report and review of literature. Int J Pediatr Otorhinolaryngol. 2012;76(4):606-8. 